\title{
Evaluating the temperature effects of ice and heat tests on ptosis due to Myasthenia Gravis
}

\author{
Emmanuel Marinos ${ }^{1} \cdot$ Katherine Buzzard $^{2} \cdot$ Clare L. Fraser $^{3} \cdot$ Stephen Reddel ${ }^{2}$
}

Received: 19 December 2017 / Revised: 5 April 2018 / Accepted: 11 April 2018 / Published online: 10 May 2018

(c) The Royal College of Ophthalmologists 2018

\begin{abstract}
Aims The aims of this study are as follows: (1) to describe a standardised methodology for the ice test, and where necessary a heat test; (2) to determine the effects of local cooling vs. heating on ptosis in myasthenia gravis (MG) patients.

Methods Twenty-three MG patients with ptosis were identified from a tertiary referral neuroimmunology clinic, seventeen with significant ptosis $\geq 2 \mathrm{~mm}$. Latex party balloons were filled with ice and cold tap water for the ice test, or with water heated to $45^{\circ} \mathrm{C}$ for the heat test. Each test was performed for $2 \mathrm{~min}$, with ruler measurement and photographs taken of the palpebral aperture before and immediately after each test.

Results This standardised method was efficient in a clinical setting while maintaining efficacy. In all 23 patients, the mean improvements in ptosis with the ice, rest, and heat tests were $2.3( \pm 1.5) \mathrm{mm}, 1.3( \pm 1.1) \mathrm{mm}$, and $0.33( \pm 1.4) \mathrm{mm}$ respectively. In the 17 patients with significant ptosis, the mean improvements in ptosis with the ice, rest, and heat tests were 2.9 $( \pm 1.2) \mathrm{mm}, 1.8( \pm 0.92) \mathrm{mm}$, and $0.83( \pm 1.4) \mathrm{mm}$, respectively. In these 17 patients, the ice test improved ptosis by $1.3 \mathrm{~mm}$ more than rest $(p<0.0001)$. The heat test improved ptosis by $1.1 \mathrm{~mm}$ less than rest $(p=0.0013)$. The ice test improved ptosis by $2.3 \mathrm{~mm}$ more than the heat test $(p<0.0001)$.

Conclusions The standardised methodology of ice and heat testing was safe, easily reproducible and effective. We confirm that temperature had a significant effect on ptosis in MG, and therefore refute the notion that measured changes are purely due to the associated rest.
\end{abstract}

\section{Background}

Myasthenia gravis (MG) is an autoimmune disease typically caused by antibodies to postsynaptic acetylcholine receptors (AChR) at the neuromuscular junction of skeletal muscle. Ptosis is the most common presenting symptom in patients with MG, present in up to 50-70\% of individuals. However, ptosis is a non-specific symptom that can be caused by other disorders such as levator aponeurosis dehiscence, Horner's syndrome, oculomotor nerve palsy, or congenital ptosis [1]. The Tensilon test (intravenous edrophonium administration) has been the traditional gold standard for diagnosing MG and more recently single fibre electromyography (SFEMG)

Emmanuel Marinos

Emmanuel.marinos@gmail.com

1 The University of Sydney. Sydney, Australia, Postal address: 6 Byron Street, Coogee, NSW 2034, Australia

2 Concord Repatriation General Hospital, Sydney, NSW, Australia

3 Macquarie University Hospital, Sydney, NSW, Australia
[2]. Other invasive tests for diagnosing $\mathrm{MG}$ include serum acetylcholine receptor antibody levels, and repetitive stimulation studies [3, 4].

The non-invasive ice test has been reported to show improvements in myasthenic ptosis with comparable sensitivities and specificities to invasive testing, without the associated risk and cost [5-7]. Others have suggested that there is no real benefit from the cooling effect of the ice itself, and the only effect of the ice test is due to the intrinsic rest of the lid during the application of ice [8]. Temperature is known to affect muscle fatigue in patients with MG $[9,10]$. One theoretical basis for this phenomenon is that acetylcholinesterase activity is decreased with cooling [11]. Additional effects on ion channel function, and/or dissociation of the transmitter-receptor complex are also plausible [12].

The methodology of the ice test has not been standardised. Direct application of ice as a block or cube of ice to the eyelid can be uncomfortable and does not cover or mould to the shape of the lid. Application of heat to the eyelid also requires a malleable mode of administration at a temperature below the risk of thermal burns. 
Fig. 1 Improvement in ptosis was greatest with ice, and least with heat, consistent with an effect of temperature over and above the intrinsic rest component. Mean differences are shown in all 23 patients (left) and in the 17 patients with significant ptosis of $\geq 2 \mathrm{~mm}$ (right). All differences were significant to $p<0.005$ as shown

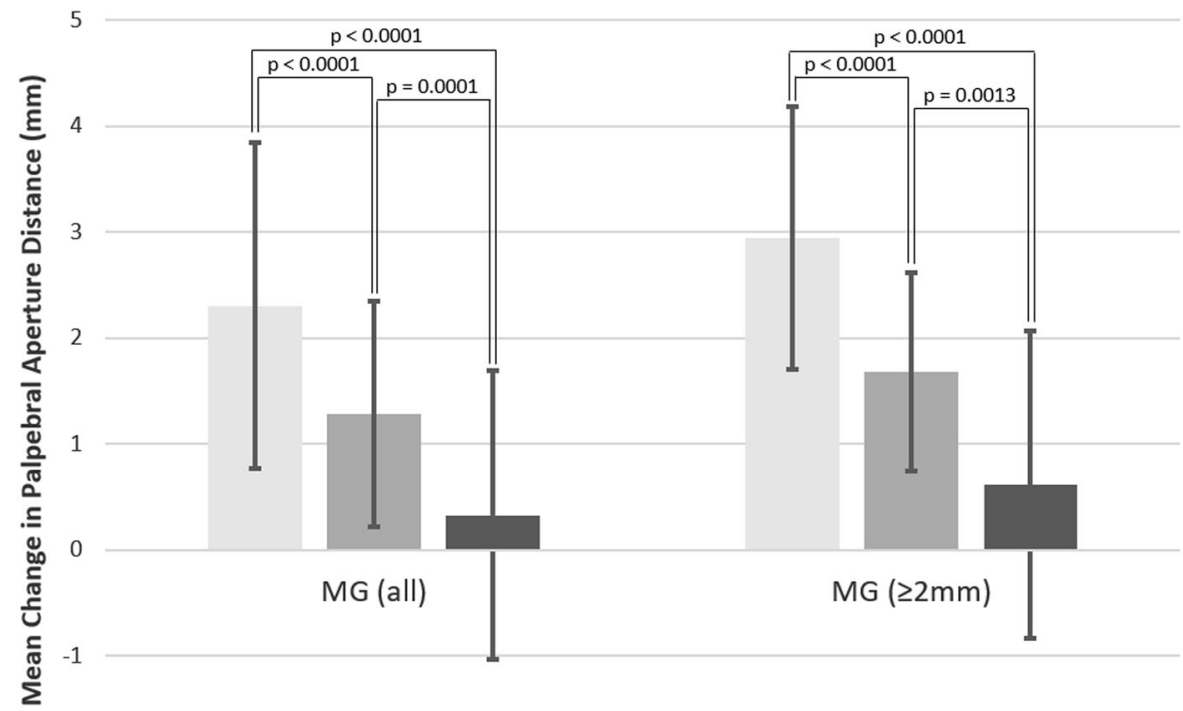

$-2$

Ice Test $\quad$ Rest Test $\square$ Heat Test
We aimed to establish a standardised, inexpensive, widely available method to administer the ice test, and to determine whether temperature has an additional effect beyond rest alone.

\section{Methods}

The study was approved by the Concord Hospital Human Research Ethics Committee (HREC/14/CRGH/123). Individual signed patient consent forms were obtained prior to testing for every patient. In addition, an optional consent form for allowing the use of identifiable photographs for publication purposes was signed by some participants.

Twenty-three patients with unilateral or bilateral ptosis due to myasthenia gravis participated in the study over a 6month period (December 2014-May 2015). Patients had a confirmed diagnosis of MG based on the detection of AChR antibodies and/or positive electrodiagnostic studies. No patients with abnormal facial sensation or impaired pain perception/awareness were included for safety reasons. Where possible, patients taking pyridostigmine bromide for MG were asked to withhold the medication for $12 \mathrm{~h}$ prior. Testing was performed during the day (between 9:00 am and 5:00 pm), with the time of testing varying between patients due to logistical restraints.

For the ice test, generic latex party balloons (Coles supermarket, Australia) had ice cubes inserted and then filled with cold water without being stretched to give an approximate temperature of $0{ }^{\circ} \mathrm{C}$. An equivalent such as a tied off nitrile examination glove should be used for those with a latex allergy. For the heat test, balloons were filled with water heated to $45^{\circ} \mathrm{C}$ (measured with a thermometer). This temperature was chosen as it was not associated with burn risk and was $10^{\circ} \mathrm{C}$ below the temperature required for a burn at a duration ten times longer than used in this study [13]. Balloons were kept until use in plastic hospital issue jugs with water and ice or $45^{\circ} \mathrm{C}$ water until use to ensure temperature stability. For the rest test, patients were positioned supine with their eyes comfortably closed for $2 \mathrm{~min}$, without forced eye closure or contraction. Tests were first performed on the investigators to ensure no undue discomfort for the patients.

The principal measurement was the palpebral aperture of the ptosed eye measured to the closest $0.5 \mathrm{~mm}$ with a ruler. The severity of the ptosis $(\geq 2 \mathrm{~mm}=$ significant $)$ was defined in unilateral ptosis by comparison to the other eye, and in bilateral ptosis as the distance during maximal attempted eye opening below the superior limbus, as previously defined [14].

In all tests, the patients were asked to lie supine. Each test was performed on the ptosed or most ptosed eyelid for a duration of $2 \mathrm{~min}$, followed by a second measurement of the palpebral aperture of the tested eye immediately after the removal of the pack. Each measurement was taken with the patients upright, with the investigator's hand pressed on the participants' forehead to minimise frontalis overactivity. The tests were completed in the same order for each patient: starting with the ice test, followed by the rest test, and finally the heat test. After each measurement, sufficient time was allowed for the ptosis to return to its baseline measurement. This did not exceed $5 \mathrm{~min}$ for any of the tests for each patient. 
Table 1 Mean change in palpebral aperture distance

\begin{tabular}{llll}
\hline & $\begin{array}{l}\text { Ice Pack mean } \Delta( \pm \text { s.d. }), \\
\mathrm{mm}\end{array}$ & $\begin{array}{l}\text { Rest Test mean } \Delta( \pm \text { s.d. }), \\
\mathrm{mm}\end{array}$ & $\begin{array}{l}\text { Heat Pack mean } \Delta( \pm \text { s.d. }), \\
\mathrm{mm}\end{array}$ \\
\hline MG (all; $n=23)$ & $2.30( \pm 1.54)$ & $1.28( \pm 1.06)$ & $0.33( \pm 1.36)$ \\
MG $\left(\geq 2 \mathrm{~mm}^{\mathrm{a}} ; n=17\right)$ & $2.94( \pm 1.24)$ & $1.68( \pm 0.93)$ & $0.62( \pm 1.45)$ \\
\hline
\end{tabular}

${ }^{\text {aa }}$ atients with significant ptosis of $\geq 2 \mathrm{~mm}$ at baseline

Statistical evaluation between paired tests was calculated with two tailed Wilcoxon signed-rank tests using GraphPad Prism (GraphPad Software, Inc. San Diego, CA, USA). This non-parametric test was used as a paired difference test between each set of data given that the samples were not assumed to be normally distributed. Significance per tail was set at $p<0.025$.

\section{Results}

23 patients with known MG participated (Fig. 1). Of these, 6 patients had mild ptosis (herein baseline level of ptosis of $<2 \mathrm{~mm}$ ), and 17 had more significant ptosis (greater than or equal to $2 \mathrm{~mm}$ ) [14]. The methodology of performing the ice and heat tests using generic latex party balloons filled with ice and water was simple, required about $10 \mathrm{~min}$, and there were no complaints of discomfort.

The results of the ice, rest, and heat tests are summarised in Table 1. In all MG patients $(n=23)$, the mean change in palpebral aperture distance due to the ice test was $1.0 \mathrm{~mm}$ greater than the rest test $(p<0.0001)$. The mean change after the ice test was $2.0 \mathrm{~mm}$ greater than the heat test $(p<0.0001)$. The mean change due to the rest test was 1.0 $\mathrm{mm}$ greater than the heat test $(p=0.0001)$. In MG patients with significant ptosis $(n=17)$, the mean change in palpebral aperture distance due to the ice test was $1.3 \mathrm{~mm}$ greater than the rest test $(p<0.0001)$. The mean change after the ice test was $2.3 \mathrm{~mm}$ greater than the heat test $(p<0.0001)$. The mean change due to the rest test was $1.1 \mathrm{~mm}$ greater than the heat test $(p=0.0013)$. All of these differences were highly significant $(p<0.005)$. An example of these differences is seen in the patient in Fig. 2.

\section{Discussion}

Myasthenia gravis (MG) is estimated to affect $10-20$ per 100,000 people, and is one of the most common treatable causes of ptosis. Over $50 \%$ of patients who initially present with isolated ocular symptoms will go on to develop generalised MG within two years [15]. Early diagnosis may reduce future morbidity through recognition of worsening MG symptoms and prompt initiation of treatment. The ice test is widely used as a simple, non-invasive, easily

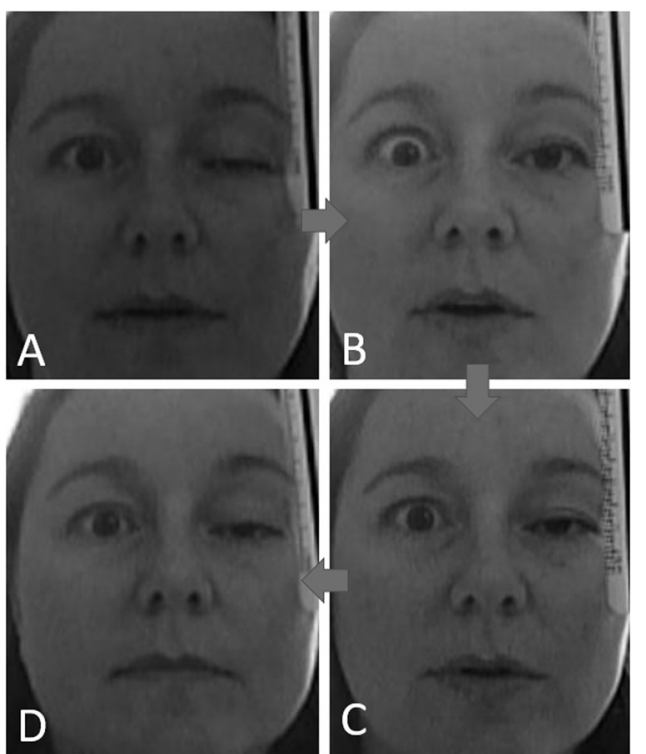

Fig. 2 A patient with significant ptosis of the left eye due to myasthenia gravis. When compared with baseline (a: Palpebral Aperture: 4 $\mathrm{mm}$ ), the ice test (b: PA: $8 \mathrm{~mm}$ ) resulted in greater increase in ptosis compared to the rest test (c: PA: $6 \mathrm{~mm})$ and heat test (d: PA: $5 \mathrm{~mm})$

interpretable diagnostic test. While the reliability of the ice test has been confirmed by several studies [5-7, 16-19], values for sensitivity and specificity have varied widely. A recent study suggested the ice test has a sensitivity of only $38.5 \%$ after a single test, and repeated tests on the same patient giving the same results $61.5 \%$ of the time [20]. However, the study noted that the ice test maintained a very high specificity $(97.4 \%)$ and positive predictive value (90.9\%) even after a single application, and that a second application of the test increased its sensitivity to $73.1 \%$ [20]. This suggests that an initial negative ice pack test should ideally be followed by a second application of the test at a later time.

The data comparing effects of ice and heat tests on myasthenic ptosis is currently limited to a single case series of 4 patients, which found that both the ice and heat tests improved ptosis of MG patients equally [8]. The authors, therefore, suggested that the relevant factor was rest, which is implicit in the ice test as the lid is closed to apply ice, rather than temperature. In our study of $23 \mathrm{MG}$ patients, the results showed marked differences in the improvement of ptosis with the 3 non-invasive tests. The ice test showed the 
greatest improvement, followed by the rest test, and finally the heat test. This suggests that local increase of temperature has a significant deleterious effect in testing of 2-min duration, albeit not completely overcoming the beneficial effect of rest. It may be that higher temperatures, not permitted by our safety parameters, would have a more deleterious effect.

In the case series by Movaghar, the results showed no difference in the improvement in ptosis with ice and heat testing, suggesting that potentially the main effect arising from these tests was due solely to the intrinsic rest component [8]. However, it is also noted that their study methods utilised pack testing of 15 min duration rather than the more commonly used 2 min duration, as used in this study. It is possible that with longer duration of testing, the rest component may ultimately overwhelm any effect of temperature. It is also possible that the number of patients in that study, 4 (four), was insufficient.

Several mechanisms have been proposed to explain the effects of temperature on the neuromuscular junction, including temperature effects on the release of acetylcholine, endplate sensitivity, and differences in the activity of acetylcholinesterase [21, 22]. Acetylcholinesterase activity has been shown to decrease by $\sim 18 \%$ in whole human blood and $38 \%$ in plasma when temperatures are decreased in vitro from 40 to $30^{\circ} \mathrm{C}$ [11]. As enzyme activity is known to decrease with cooling, even moderate cooling of the neuromuscular junction is thought to significantly decrease the activity of acetylcholinesterases, thereby potentiating neuromuscular transmission [21]. However, other effects including on the acetylcholine receptor ion channel function, and/or dissociation of the transmitter-receptor complex have also been suggested [12].

If a clinical test is to be regarded as validated for diagnostic use, we suggest it also needs to be standardised, measurable and recordable for later review, as is generally the case with electrophysiological and antibody tests for myasthenia. The method used in this study is standardised, requires no special equipment, is measurable, and using a camera is recordable and able to be printed or transferred to an electronic medical record for later review. This also allows for better comparison between studies in the future.

The main limitations of this study were the lack of blinding and the lack of independent recordable assessment of results. As the primary investigator and patient were not blinded to the interventions, there is a theoretical potential for confirmation bias. The other major limitation of the study's methodology was the assessment of results. For logistic reasons, each study was conducted by a single investigator, including the ruler measurement of results first, and then taking photographs with the patient holding the ruler. It was difficult to immediately obtain adequate photographs as the ruler was often poorly positioned, and furthermore the improvement began to worsen again over time. This meant that the plan to have an independent observer measurement from the photographs was not possible. This is certainly a potential source of results bias, as a full independent scoring of results was not possible. For future studies, a more rigorous approach would have a clearly measurable photograph as the initial and only measurement, perhaps calibrated with a ruler printed on sticky labels horizontally placed on the forehead to minimise tangential foreshortening. Applying ice, rest and heat testing to ptosis from other pathological causes would be of interest, but is not the subject of this paper.

\section{Conclusion}

Local cooling was shown to have a beneficial effect on improving ptosis separate to and beyond rest alone. Furthermore, local heating was shown to have a detrimental effect on ptosis compared with rest alone. The ice test was the most effective of the three tests, and as such we recommend the optimal first-line investigation of ptosis be an ice test of 2-min duration, which can be performed as a standardised test safely and simply using a generic latex party balloon filled with ice and water.

\section{Summary}

\section{What was known before}

- Theoretical benefit of cooling in myasthenia.

- Efficacy of ice and rest tests in myasthenic ptosis.

- No difference shown between ice and heat tests of 15min duration (Movaghar, 2000).

\section{What this study adds}

- Significant benefit of cooling above and beyond rest effect and worsening of heat compared with rest in short duration testing of myasthenic ptosis.

- Larger cohort studied compared to previous case series.

- Comparison of all 3 tests together.

- Evidence for benefit of cooling and detrimental effect of heat in ptosis due to MG. Shows that the 2-min ice test is the ideal non-invasive test of choice in ptosis.

\section{Compliance with ethical standards}

Conflict of interest The authors declare that they have no conflict of interest. 


\section{References}

1. Rajasekharan C, Anishkumar V, Suresh MK. Ice pack test: is it obsolete? BMJ Case Reports. 2011. https://doi.org/10.1136/bcr. 04.2011 .4066

2. Seybold ME. The office Tensilon test for ocular myasthenia gravis. Arch Neurol. 1986;43:842-3.

3. Oh SJ, Kim DE, Kuruoglu R, Bradley RJ, Dwyer D. Diagnostic sensitivity of the laboratory tests in myasthenia gravis. Muscle Nerve. 1992;15:720-4.

4. Meriggioli MN, Sanders DB. Myasthenia gravis: diagnosis. Semin Neurol. 2004;24:31-9.

5. Ertas M, Arac N, Kumral K, Tuncbay T. Ice test as a simple diagnostic aid for myasthenia gravis. Acta Neurol Scand. 1994;89:227-9.

6. Golnik KC, Pena R, Lee AG, Eggenberger ER. An ice test for the diagnosis of myasthenia gravis. Ophthalmology. 1999;106:1282-6.

7. Kubis KC, Danesh-Meyer HV, Savino PJ, Sergott RC. The ice test versus the rest test in myasthenia gravis. Ophthalmology. 2000;107:1995-8.

8. Movaghar M, Slavin ML. Effect of local heat versus ice on blepharoptosis resulting from ocular myasthenia. Ophthalmology. 2000;107:2209-14.

9. Borenstein S, Desmedt JE. Local cooling in myasthenia. Improvement of neuromuscular failure. Arch Neurol. 1975;32:152-7.

10. Mermier CM, Schneider SM, Gurney AB, Weingart HM, Wilmerding MV. Preliminary results: effect of whole-body cooling in patients with myasthenia gravis. Med Sci Sports Exerc. 2006;38:13-20.

11. Reiner E, Buntic A, Trdak M, Simeon V. Effect of temperature on the activity of human blood cholinesterases. Arch Toxicol. 1974;32:347-50.
12. Desmedt JE. How to validate Mysthenia Gravis in the patient with a diagnostic problem. In: Grob D, editor. Myasthenia Gravis: pathophysiology and management. New York (NY): New York Academy of Sciences; 1981. p. 595-8. .

13. Moritz AR, Henriques FC. Studies of thermal injury: ii. the relative importance of time and surface temperature in the causation of cutaneous burns. Am $\mathrm{J}$ Pathol. 1947;23:695-720.

14. Small RG, Sabates NR, Burrows D. The measurement and definition of ptosis. Ophthalmic Plast Reconstr Surg. 1989;5:171-5. 1

15. Kupersmith MJ, Latkany R, Homel P. Development of generalized disease at 2 years in patients with ocular myasthenia gravis. Arch Neurol. 2003;60:243-8.

16. Czaplinski A, Steck AJ, Fuhr P. Ice pack test for myasthenia gravis. A simple, noninvasive and safe diagnostic method. J Neurol. 2003;250:883-4.

17. Larner AJ. The place of the ice pack test in the diagnosis of myasthenia gravis. Int J Clin Pract. 2004;58:887-8.

18. Reddy AR, Backhouse OC. "Ice-on-eyes", a simple test for myasthenia gravis presenting with ocular symptoms. Pract Neurol. 2007;7:109-11.

19. Chatzistefanou KI, Kouris T, Iliakis E, Piaditis G, Tagaris G, Katsikeris N, et al. The ice pack test in the differential diagnosis of myasthenic diplopia. Ophthalmology. 2009;116:2236-43.

20. Park JY, Yang HK, Hwang JM. Diagnostic value of repeated ice tests in the evaluation of ptosis in myasthenia gravis. PLoS ONE. 2017; 12:e177078.

21. Rutkove SB. Effects of temperature on neuromuscular electrophysiology. Muscle Nerve. 2001;24:867-82.

22. Gutmann L. Heat exacerbation of myasthenia gravis. Neurology. 1978;28:398. 